\title{
Desarrollo léxico en la adolescencia: Un análisis de sustantivos en narraciones orales y escritas
}

\author{
Lexical development during adolescence: An analysis of nouns in \\ oral and written narratives
}

\author{
Karina Hess Zimmermann ${ }^{1}$ \\ Universidad Autónoma de Querétaro, México
}

\begin{abstract}
Resumen. Durante los años escolares la adquisición del léxico está directamente relacionada con la experiencia que los individuos tienen con la lengua escrita. En este trabajo se observó cómo adolescentes realizan una elección léxica en dos contextos discursivos diferentes: narraciones orales y escritas. Los participantes fueron 24 jóvenes pertenecientes a dos grupos sociales distintos, determinados por el acceso previo que tenían a la cultura escrita. Los textos narrativos fueron analizados con la finalidad de encontrar la presencia de elementos de lenguaje académico literario, para lo que se recurrió a un análisis del contenido semántico-pragmático de los sustantivos de acuerdo con la escala nominal de Ravid (2006). Los resultados señalan diferencias entre grupos y modalidad oral/escrito en los aspectos estudiados.
\end{abstract}

Palabras clave: desarrollo lingüístico tardío, lenguaje en la escuela, elección léxica, narraciones, sustantivos.

Abstract. During the school years, lexical acquisition is directly related to experience with written language. In this study we investigated how adolescents make lexical choices in two different discursive contexts: oral and written narratives. Participants were 24 adolescents that belonged to two different social environments, determined by the amount of previous experience with literacy. Narratives were analyzed for the presence of literary academic language. Therefore a semantic-pragmatic analysis of nouns using the Noun Scale Analysis (Ravid, 2006) was conducted. Results show differences between groups of adolescents and between oral and written modalities.

Key words: Later language development, language in schools, lexical choice, narratives, nouns.

\footnotetext{
${ }^{1}$ Karina Hess Zimmermann, Facultad de Psicología, Universidad Autónoma de Querétaro, México. E-mail: karinahess@hotmail.com Dirección postal: Jefatura de Posgrado, Facultad de Psicología, Universidad Autónoma de Querétaro, Cerro de las Campanas S/N, 76000 Querétaro, QRO. México.
} 


\section{Introducción}

En años recientes el estudio del desarrollo lingüístico tardío, es decir, aquel que se da durante los años escolares y hasta la adultez temprana, ha empezado a cobrar mayor relevancia (Barriga, 2002; Berman, 2004a; Hess, 2010; Nippold, 2007). En gran medida esto se debe al hecho de que diversas investigaciones han mostrado que las habilidades lingüísticas que se adquieren durante los años escolares están relacionadas de manera sustancial con el logro académico y vocacional, así como con la satisfacción personal de los individuos durante toda su vida.

En términos generales, se reconoce que el desarrollo lingüístico tardío involucra la adquisición de habilidades diversas que se llevan a cabo en todos los niveles del lenguaje (Barriga, 2002; Tolchinsky, 2004). Así, se ha descubierto que a partir de los seis años de edad surgen estructuras sintácticas más complejas, así como la capacidad para razonar sobre los elementos gramaticales de la lengua (Berman, 2004a; Berman \& Ravid, 2010; Gombert, 1992; Ravid, 2011). A su vez, en lo que se refiere al desarrollo pragmático, durante los años escolares aprendemos a utilizar el lenguaje de forma cada vez más pertinente y adecuada a la situación comunicativa en la que nos encontramos (Melzi \& Ely, 2010). Por otro lado, durante la infancia tardía los niños siguen adquiriendo habilidades lingüísticas y cognitivas que les permiten comprender y producir de manera más eficiente diferentes tipos de discurso (narraciones, descripciones, explicaciones, argumentaciones, entre otros) (Alvarado, Calderón, Hess \& Vernon, 2011). Adicionalmente, en el ámbito de la semántica se desarrollan diversas habilidades durante los años escolares. Por una parte, aparecen nuevos matices de significado para las palabras adquiridas en etapas anteriores, puesto que los contenidos se enriquecen y se establecen interrelaciones más complejas entre los significados (Barriga, 2002; Nippold, 2007). Además, surge la capacidad para comprender y producir expresiones polisémicas, así como no literales, como es el caso de los refranes, las metáforas, la ironía y los chistes, entre otras (Calderón, 2012; Nippold, 2007; Pence \& Justice, 2008). Finalmente, durante los años escolares y la adolescencia se da un desarrollo importante en términos del léxico, cuestión que será de interés para el presente trabajo.

Estudiar la adquisición del léxico durante la infancia tardía y la adolescencia resulta importante por diversas razones. Por un lado, sabemos que el conocimiento del léxico predice el logro académico de los niños y juega un papel esencial en su desarrollo cognitivo (Dockrell \& Messer, 2004). Uno de los aspectos más importantes que los maestros toman en cuenta para evaluar el desempeño de los alumnos se relaciona con la diversidad de palabras que estos emplean y con los usos que les dan (Beck, McKeown, \& Kucan, 2008). Adicionalmente, múltiples estudios plantean que existe una relación estrecha entre la cantidad de vocabulario que un individuo posee y su comprensión lectora: los niños con mayor vocabulario comprenden más lo que leen; a su vez, los buenos lectores adquieren un vocabulario más amplio que los lectores menos competentes (Baumann, 2008; Beck, McKeown \& Kucan, 2008; Nippold, 2007).

Durante el desarrollo lingüístico tardío se da un crecimiento muy importante en términos de vocabulario. Diversos estudios calculan que un niño promedio de seis años ingresa a la escolaridad formal con un léxico de entre 6000 y 10000 palabras y terminará sus estudios 
preuniversitarios con un vocabulario de alrededor de 40000-60000 palabras (Dockrell \& Messer, 2004; Irvin, 1990; Nippold, 2007). No todos los niños ingresan a la escuela con el mismo bagaje léxico, lo que puede representar una desventaja académica para algunos en relación con sus pares (Beck, McKeown \& Kucan, 2008; Carmiol, Ríos \& Sparks, en prensa; Mancilla-Martínez \& Lesaux, 2011).

Pero si bien existe un crecimiento muy importante en la cantidad de palabras nuevas que adquiere el individuo durante los años escolares, el proceso de adquisición léxica no solo se refiere a la acumulación de nuevos vocablos en el repertorio. Es importante señalar que durante el desarrollo lingüístico tardío los niños y adolescentes adquieren además de las palabras mismas, todo un conocimiento sobre las características y funciones que estas tienen como unidades comunicativas en el contexto en que aparecen (Cassany, Luna, \& Sanz, 1994). Es decir, los individuos deben aprender las palabras, los conceptos a los que se refieren y además las estrategias para usarlas con eficacia y adecuación.

Uno de los aspectos del léxico que los niños y adolescentes deberán adquirir durante los años escolares consiste en elegir las palabras adecuadas que respondan a un contexto situacional determinado y a un uso discursivo específico. El desarrollo de este aspecto, comúnmente denominado elección léxica, tomará al individuo muchos años (Jisa, 2004; Nippold, 2007). Ahora bien, la manera en que los niños aprenden a realizar una adecuada elección léxica puede estudiarse en diversos ámbitos del lenguaje, pero uno de los que se ofrece como una herramienta adecuada es la narración. Dado que la narración supone la adquisición de habilidades lingüísticas, cognitivas y sociales tan diversas, resulta un campo fértil para el estudio de la manera en que los diversos niveles lingüísticos se interrelacionan durante el desarrollo lingüístico (Berman, 2004b; Barriga, 2002; Hess, 2010; Hickmann, 2003). En este caso, el estudio de la elección léxica no es la excepción.

Cuando alguien construye una narración debe seleccionar las palabras adecuadas que le permitan dar mejor cuenta de los eventos narrados, así como de su opinión personal sobre los mismos (evaluación). Adicionalmente, el contexto situacional donde se encuentra el narrador tendrá incidencia sobre el tipo de palabras que emplee. De esta manera, utilizará cierto léxico cuando narre en una situación informal -como podría ser una conversación cotidiana con un amigo-, pero usará un léxico más literario o académico cuando se enfrente a la tarea de construir una narración por escrito -por ejemplo un cuento, una crónica o una noticia en el ámbito escolar-. En este sentido, un individuo lingüísticamente competente será aquel que pueda adaptar su lenguaje a una diversidad de circunstancias comunicativas por medio de una diversificación de medios lingüísticos (Tolchinsky, Rosado, Aparici \& Perera, 2005). Será tema del presente estudio, por tanto, describir la manera en que adolescentes de dos entornos sociales distintos eligen las palabras en dos tareas narrativas: narración oral y narración escrita.

\section{El léxico en el medio escolar}

Cuando un niño ingresa a un ámbito escolarizado más formal se encuentra con un nuevo tipo de vocabulario. En términos generales los estudios señalan que las palabras a las que se enfrentan los niños durante los años escolares son más abstractas y menos transparentes semánticamente que aquellas a las que anteriormente estaban acostumbrados en las interacciones cotidianas (Irvin, 1990; Nippold, 
2007; Snow \& Uccelli, 2009; Zwiers, 2008). Esto se debe a que el vocabulario escolar obedece en gran medida a un sistema diferente: la lengua escrita.

La lengua escrita, dadas sus características, tiende a emplear un léxico más específico, especializado, semánticamente diverso y abstracto que la lengua oral (Schleppegrell, 2004; Ravid \& Cahana-Amitay, 2005; Snow \& Uccelli, 2009). Además, incluye un vocabulario más complejo que obedece a la expresión de conceptos e ideas nuevas, así como palabras cuya comprensión requiere del manejo de habilidades de morfología derivativa (Larsen \& Nippold, 2007; Nippold, 2007; Nippold \& Sun, 2008; Ravid, 2006; 2009). A su vez, se ha observado que la lengua escrita contiene palabras más largas y una mayor diversidad de palabras que la lengua oral (Barton, 2007), así como un registro que involucra un uso del léxico que marca un distanciamiento entre escritor y lector (Ravid \& Cahana-Amitay, 2005; Schleppegrell, 2004). Por otro lado, en la lengua escrita el niño debe aprender a emplear el lenguaje sin ayuda de un contexto externo que apoye la construcción del significado, lo que lo llevará a establecer relaciones más complejas entre significados. De manera adicional, la lengua escrita tiende a ser más condensada y a incluir estructuras sintácticas más densas, es decir, una mayor cantidad de información en una menor cantidad de palabras con mayor carga conceptual (Berman \& Ravid, 2010; Ravid \& Cahana-Amitay, 2005; Tolchinsky, Rosado, Aparici \& Perera, 2005).

Por todo lo anterior, el uso de vocabulario técnico, especializado, abstracto y genérico, característico de la lengua escrita, ha sido documentado como de adquisición tardía (Barriga, 2002; Jisa, 2004; Nippold, 2007; Ravid \& Cahana-Amitay, 2005; Schleppegrell,
2004). Dicha adquisición además ha sido asociada directamente con la pertenencia del individuo a una cultura escrita y con la cantidad de experiencia que tenga con cierto tipo de vocabulario (Dockrell \& Messer, 2004; Nippold, 2007; Ramachandra \& Karanth, 2007). Por tanto, la exposición al vocabulario propio de la lengua escrita en el ámbito escolarizado tendrá una importante incidencia en la apropiación de un léxico diferente al de la oralidad. De manera adicional, diversos estudios señalan que el hogar también juega un papel importante en la adquisición de habilidades lingüísticas propias de la lengua escrita (al respecto véase Hess, 2010) y que los niños que provienen de hogares en donde la cultura escrita es valorada presentan mayor vocabulario y mayores posibilidades para organizar su discurso.

\section{El léxico académico}

Uno de los mayores retos en el desarrollo lingüístico tardío es la apropiación de un lenguaje académico (Jisa, 2004; Nippold, 2007; Schleppegrell, 2004; Snow \& Uccelli, 2009; Zwiers, 2008). Este involucra el uso especializado de palabras, estructuras sintácticas y géneros discursivos que le permitan al individuo conocer, pensar, leer y escribir en diversos ámbitos académicos. En términos generales, el lenguaje académico requiere de la apropiación de cuatro tipos de lenguaje especializado: lenguaje matemático, lenguaje científico, lenguaje de las ciencias sociales y lenguaje literario (Zwiers, 2008). Dado que el presente trabajo versa sobre narraciones, se centrará en el desarrollo del lenguaje académico de tipo literario.

El lenguaje académico pertenece a un ámbito social al que los niños comúnmente tienen poco acceso durante los primeros años de vida, dado que no es común en las interacciones cotidianas. 
El lenguaje académico se distingue de otras manifestaciones lingüísticas en varios aspectos. Por una parte, cumple con la función de permitir describir conceptos abstractos de una manera más clara (Pence \& Justice, 2008; Zwiers, 2008), lo que lo convierte en una herramienta importante para el lenguaje científico. Además, facilita la expresión de procesos cognitivos complejos o de alto nivel (higher-order) (Dockrell \& Messer, 2004; Nippold, 2007; Zwiers, 2008) y presenta la información de una manera más densa, objetiva e imparcial (Mostacero, 2004; Schleppegrell, 2004; Snow \& Uccelli, 2009). Todas estas funciones del lenguaje académico implican que requiera de una contextualización más explícita, en la medida en que se emplea con un propósito y en una situación y cultura específicos. A la vez, es un tipo de lenguaje más demandante cognitivamente para el individuo, dado que es menos familiar y automático y a la vez más condensado en términos de manejo de información (Schleppegrell, 2004). Es por ello que el dominio del lenguaje académico involucra el desarrollo de habilidades lingüísticas, cognitivas y socioculturales/psicológicas complejas (Snow \& Uccelli, 2009; Ravid \& Cahana-Amitay, 2005).

Debido a que el lenguaje académico conlleva la expresión de significados diferentes y responde a contextos lingüísticos distintos de los de la oralidad, hace uso de recursos léxicos, gramaticales y discursivos más complejos. Schleppegrell (2004), Snow y Uccelli (2009) y Zwiers (2008) señalan que el lenguaje académico posee diversas características lingüísticas. En primer lugar, tiende a presentar una organización discursiva más jerárquica y estructurada. A su vez, dicha organización textual tiende a marcarse explícitamente mediante el uso de marcadores textuales (al respecto véase también Hyland, 2005). En el lenguaje académico existe una enorme carga informativa, puesto que hay una mayor densidad léxica (abundan las nominalizaciones, las expresiones más formales y prestigiosas, así como el uso de términos abstractos y técnicos) y una mayor complejidad sintáctica (oraciones más largas y con una mayor cantidad de subordinación). Adicionalmente, es muy frecuente el uso de expresiones de lenguaje figurado -en gran medida para describir conceptos abstractos-, así como de marcas de modalización (uso de verbos y adverbios que permitan señalar intención, obligación, probabilidad y condicionalidad, entre otros).

Una de las formas en las que es posible estudiar cómo los adolescentes se apropian de un lenguaje académico es ver si empiezan a incluir términos más técnicos, especializados y abstractos en los contextos que así lo requieren (contextos más formales, académicos, más cercanos a los de la lengua escrita). Analizar si son capaces de realizar una elección léxica de acuerdo con el contexto al que se enfrentan -en el caso de este estudio un contexto de narración oral informal y otro de narración escrita más de tipo literario-, nos permite ver si han adquirido la habilidad para expresarse de manera distinta dependiendo del género lingüístico al que se enfrentan. En este sentido, el análisis de la manera en que eligen usar los sustantivos es una excelente opción, puesto que se ha documentado que este tipo de análisis permite dar cuenta del conocimiento y uso lingüísticos durante el proceso de adquisición lingüística en las etapas tardías (Ravid, 2006). Ello se debe a que los sustantivos desempeñan un papel crucial en la organización discursiva porque conllevan en gran medida el flujo de información textual (Ravid \& Cahana-Amitay, 2005). A su vez, en el análisis de los sustantivos es posible ver dos aspectos importantes del lenguaje académico y, por tanto, del lenguaje 
académico literario: las nominalizaciones y el uso del sentido figurado. Las nominalizaciones son interesantes de analizar porque tienden a condensar explicaciones largas en pocas palabras. Esto implica en el individuo un mayor procesamiento cognitivo (más ideas por cláusula, mayor abstracción y la necesidad de mantener la pista de las diversas relaciones abstractas que se establecen entre las diferentes entidades del texto). Por otra parte, los sustantivos usados en sentido figurado nos permiten ver cómo los niños y adolescentes se apropian de una nueva herramienta lingüística para expresar conceptos complejos y abstractos.

Con base en lo expuesto anteriormente, el objetivo del presente trabajo fue observar la presencia de elementos del lenguaje académico literario en dos tipos de textos narrativos (narraciones personales orales y escritas) de adolescentes de dos entornos sociales distintos. De manera específica se busca describir la manera en que los jóvenes hacen un uso diferenciado de los sustantivos (elección léxica) en ambos tipos de texto. Para ello se recurre a un análisis del contenido semántico-pragmático de los sustantivos de acuerdo con la escala nominal de Ravid (2006).

\section{Método}

\section{Participantes}

En el estudio participaron 24 adolescentes de clase media de 12, 14 y 16 años que vivían en la ciudad de Querétaro, México. Dado que se ha señalado que el entorno social en el que se desenvuelven los individuos -y de manera directa la pertenencia a una cultura escritaincide en el desarrollo lingüístico tardío, fueron seleccionados jóvenes de dos entornos sociales diferentes, determinados por un mayor o menor acceso a la lengua escrita en la escuela y el hogar. De esta manera, los adolescentes del Grupo 1 asistían a una escuela privada donde se realizaba un trabajo muy intenso con la lengua escrita (talleres de investigación, proyectos de creación literaria, visitas semanales a la biblioteca, periódico escolar, entre otros) y provenían de hogares donde la lengua escrita jugaba un papel importante dentro de las actividades cotidianas familiares. En cambio, los jóvenes del Grupo 2 asistían a una escuela privada en donde se priorizaba el desarrollo de habilidades académicas distintas a las de la lengua escrita (computación, tecnologías, proyectos de matemáticas) y reportaron que en su familia la lectura y escritura no jugaban un rol primordial. Adicionalmente, los dos grupos fueron balanceados por género. La Tabla 1 muestra las características de los participantes del estudio.

Obtención de los datos

Todos los participantes fueron entrevistados de manera individual en un salón que las escuelas proporcionaron para el estudio. Para la obtención de las narraciones personales orales se recurrió al método propuesto por Labov y Waletzky (1997), que consiste en preguntar a los participantes si alguna vez han estado en peligro de muerte. Durante la narración el investigador no hizo preguntas al participante y sus intervenciones consistieron únicamente en hacer comentarios generales como ¿ah, sí?, entiendo y similares.

Tabla 1

Participantes del estudio

\begin{tabular}{lcccc}
\hline & \multicolumn{2}{c}{ Grupo 1 } & \multicolumn{2}{c}{ Grupo 2 } \\
\cline { 2 - 5 } & Hombres & Mujeres & Hombres & Mujeres \\
\hline 12 años & 4 & 4 & 4 & 4 \\
14 años & 4 & 4 & 4 & 4 \\
16 años & 4 & 4 & 4 & 4 \\
\hline
\end{tabular}


Durante la conversación se procuró obtener la mayor cantidad de narraciones posibles de cada participante. En términos generales todos los adolescentes relataron entre tres y cuatro situaciones. Posteriormente, con la finalidad de obtener las narraciones escritas, se les pidió a los jóvenes que eligieran el relato que consideraran el más interesante para ser incluido en un libro de historias para adolescentes. Ya que lo habían elegido, se les pedía que lo pusieran por escrito.
Análisis de los datos

Para el análisis fueron tomadas en cuenta tanto la narración oral elegida por cada participante como su contraparte escrita. Todas las narraciones (orales y escritas) fueron transcritas bajo el sistema CHILDES (MacWhinney, 1991), para lo cual fueron segmentadas por cláusulas de acuerdo con la propuesta de Berman y Slobin (1994). A continuación se procedió a categorizar todos los sustantivos presentes

Tabla 2

Categorías de Sustantivos de Acuerdo con la Escala Nominal

\begin{tabular}{|c|c|c|}
\hline Orden & Descripción & Ejemplos \\
\hline \multicolumn{3}{|c|}{ Entidades de Primer Orden } \\
\hline Nivel 1 & $\begin{array}{l}\text { Objetos concretos o personas } \\
\text { específicas en el texto }\end{array}$ & $\begin{array}{l}\text { pelota, bicicleta, puerta, un niño de mi } \\
\text { clase }\end{array}$ \\
\hline Nivel 2 & $\begin{array}{l}\text { Nombres propios para designar } \\
\text { individuos }\end{array}$ & Juan, Pedro \\
\hline Nivel 3 & $\begin{array}{l}\text { Sustantivos referentes a colectivos } \\
\text { propios de una locación }\end{array}$ & $\begin{array}{l}\text { orquesta, escuela, comunidad, } \\
\text { instituto, biblioteca, campamento }\end{array}$ \\
\hline Nivel 4 & Sustantivos de roles sociales & $\begin{array}{l}\text { primo, vecino, alumno, novio, director, } \\
\text { compañero }\end{array}$ \\
\hline Nivel 5 & $\begin{array}{l}\text { Genéricos (sustantivos y pronombres } \\
\text { con función genérica) }\end{array}$ & niños (gen), personas, alguien, gente \\
\hline \multicolumn{3}{|c|}{ Entidades de Segundo Orden } \\
\hline Nivel 6 & $\begin{array}{l}\text { Sustantivos que designan unidades } \\
\text { temporales }\end{array}$ & $\begin{array}{l}\text { (hace medio) año, (cuando estaba en) } \\
\text { cuarto, (un) día }\end{array}$ \\
\hline Nivel 7 & Sustantivos de eventos con duración & fiesta, juego, pelea, terapia \\
\hline Nivel 8 & $\begin{array}{l}\text { Sustantivos abstractos imaginables o } \\
\text { de metáforas cristalizadas }\end{array}$ & $\begin{array}{l}\text { gritos, golpes, respuestas, rasguño, } \\
\text { (mantener bajo) control }\end{array}$ \\
\hline \multicolumn{3}{|c|}{ Entidades de Tercer Orden } \\
\hline Nivel 9 & $\begin{array}{l}\text { Sustantivos abstractos no imaginables } \\
\text { y poco individuados }\end{array}$ & carácter, asunto, meta, estilo \\
\hline Nivel 10 & $\begin{array}{l}\text { Sustantivos abstractos derivados de } \\
\text { verbos, adjetivos y otros sustantivos }\end{array}$ & $\begin{array}{l}\text { negación, discusión, comportamiento, } \\
\text { prejuicio, tolerancia }\end{array}$ \\
\hline
\end{tabular}

Nota. Adaptado de "Semantic development in textual contexts during the school years: Noun Scale analyses," por D. Ravid (2006). 
en los relatos con base en la escala nominal de Ravid (2006), que ha sido empleada para el análisis de textos narrativos y expositivos en niños y adultos. De acuerdo con la autora, los sustantivos pueden ser categorizados con base en su contenido semántico-pragmático en tres órdenes y, a su vez, en diez categorías diferentes que van desde los sustantivos más concretos hasta los más abstractos. Bajo este enfoque, los sustantivos del primer orden corresponden a todos los sustantivos concretos, nombres propios, nombres para lugares o roles sociales, así como a los genéricos referentes a personas y cosas en general. Por último, los sustantivos de tercer orden son aquellos sustantivos abstractos que se encuentran fuera del espacio y tiempo. La Tabla 2 resume las categorías en las que se clasificaron los sustantivos.

\section{Resultados}

Todos los análisis se hicieron con la finalidad de observar si existían diferencias debidas al grupo al que pertenecían los participantes (Grupo 1 y Grupo 2), a la edad de los mismos o a la modalidad oral/escrito. Es importante resaltar que en términos generales no se observaron diferencias importantes debidas a la edad, por lo que solo se presentan resultados con respecto a las diferencias observadas entre grupos y modalidades oral/escrito.

\section{Análisis de la densidad nominal}

El primer análisis consistió en ver si existían diferencias en la densidad nominal de las cláusulas, es decir, en la cantidad de sustantivos totales presentes por cláusula. La Tabla 3 presenta los resultados de este análisis.

En términos generales se observa que, como era de esperarse, para ambos grupos existe una mayor densidad nominal en las narraciones
Tabla 3

Promedio de Densidad Nominal por Grupo y Modalidad

\begin{tabular}{lcc}
\hline & $\begin{array}{c}\text { Narraciones } \\
\text { orales }\end{array}$ & $\begin{array}{c}\text { Narraciones } \\
\text { escritas }\end{array}$ \\
\hline Grupo 1 & .78 & 1.19 \\
Grupo 2 & .89 & 1.09 \\
\hline
\end{tabular}

escritas que en las orales. Esto indica que los relatos escritos poseen una mayor cantidad de información en menos cláusulas, lo que nos habla de un mayor empaquetamiento de información en la escritura. A su vez, se observa que el Grupo 1 tiene una densidad nominal ligeramente mayor que el Grupo 2 en las dos modalidades.

\section{Análisis por tipo de sustantivos}

A continuación se procedió a analizar la densidad nominal pero tomando en cuenta el tipo de sustantivos presentes. Así, se realizó un análisis de la cantidad de sustantivos categorizados bajo los tres tipos de órdenes (primer, segundo y tercer orden) que se encontraban en las cláusulas para los dos tipos de narración en los dos grupos. La Tabla 4 presenta los resultados de este análisis.

En la Tabla 4 se observa cómo en los dos grupos muestran un comportamiento diferente entre sí. El Grupo 1 presenta para ambas modalidades (oral/escrito) una mayor cantidad de sustantivos de primer orden, es decir, de aquellos que se refieren a entidades concretas, seguida por la presencia de sustantivos de segundo orden, que denotan eventos o procesos observables que se dan en un tiempo determinado. Los sustantivos del tercer orden, es decir, aquellos que se refieren a entidades abstractas y poco imaginables fueron mucho menos frecuentes dentro de las cláusulas. Además, se observa que en todos los casos hay una mayor densidad en la modalidad 
Tabla 4

Promedio de Densidad Nominal por Tipo de Sustantivo (Primer, Segundo y Tercer Orden) para los Dos Grupos en Ambas Modalidades

\begin{tabular}{lcccccc}
\hline \multirow{2}{*}{ Tipo de sustantivo } & \multicolumn{2}{c}{ Grupo 1 } & & \multicolumn{2}{c}{ Grupo 2 } \\
\cline { 2 - 3 } \cline { 6 - 7 } & Oral & Escrito & & Oral & Escrito \\
\hline Primer orden & .48 & .56 & & .37 & .37 \\
Segundo orden & .27 & .46 & & .55 & .37 \\
Tercer orden & .08 & .30 & & .02 & .03 \\
\hline
\end{tabular}

escrita que en la oral. En cambio, en el Grupo 2 no se observan diferencias importantes entre la modalidad oral/escrito, además de que los sustantivos de segundo orden en la oralidad fueron los más frecuentes. Estos resultados apuntan a que existe un comportamiento distinto entre los dos grupos analizados en términos del tipo de sustantivos empleados para cada una de las modalidades (oral/escrito).

Con la finalidad de analizar un poco más a fondo las diferencias entre los grupos en el tipo de sustantivos empleados, se procedió a analizar la proporción de sustantivos por tipo en el total de sustantivos. La Tabla 5 resume los resultados obtenidos.

En la Tabla 5, para ambos grupos y modalidades existe una predominancia de los sustantivos de primer orden, seguida por una de los de segundo orden. Por su parte, los sustantivos del tercer orden son los menos frecuentes, cuestión que es de esperarse dado que, como se recordará, se trata de los sustantivos que involucran mayor complejidad.

En una comparación por modalidades se muestra para ambos grupos de adolescentes que la lengua oral presenta una predominancia de sustantivos de primer orden, en tanto que la lengua escrita favorece la aparición de sustantivos de segundo y tercer orden. Esto también es de esperarse, dado que la literatura reporta que la lengua escrita favorece la aparición de sustantivos de mayor complejidad. Sin embargo, en el uso de sustantivos de tercer orden es importante la diferencia entre los dos grupos de adolescentes. Mientras que los jóvenes del Grupo 2 presentan un porcentaje muy bajo de este tipo de sustantivos en sus narrativas $(1 \%$ en las orales y $2 \%$ en las escritas), los adolescentes del Grupo 1 presentan más en la oralidad (5\%) que los del Grupo 2 y duplican su uso en la lengua escrita $(12 \%)$. Esto lleva a pensar que las experiencias a las que se han enfrentado los adolescentes del Grupo 1

Tabla 5

Proporción de Tipos de Sustantivos (Primer, Segundo y Tercer Orden) en el Total de Sustantivos para Ambos Grupos y Modalidades

\begin{tabular}{lccccc}
\hline \multirow{2}{*}{ Tipo de sustantivo } & \multicolumn{2}{c}{ Grupo 1} & & \multicolumn{2}{c}{ Grupo 2} \\
\cline { 2 - 3 } \cline { 6 - 7 } & Oral & Escrito & & Oral & Escrito \\
\hline Primer orden & $70 \%$ & $60 \%$ & & $72 \%$ & $64 \%$ \\
Segundo orden & $24 \%$ & $28 \%$ & & $27 \%$ & $34 \%$ \\
Tercer orden & $5 \%$ & $12 \%$ & & $1 \%$ & $2 \%$ \\
\hline
\end{tabular}

Actualidades en Psicología, 27(115), 2013, 113-127 
con la lengua escrita los han llevado a conocer y emplear una mayor cantidad de sustantivos de tercer orden.

\section{Análisis de los sustantivos de tercer orden}

Dados los resultados anteriores, se decidió hacer un análisis más profundo de la manera en que los adolescentes de ambos grupos hacen uso de los sustantivos de tercer orden (abstractos no imaginables poco individuados y abstractos derivados de otras categorías gramaticales). Para ello se buscaron en primer lugar todos los sustantivos de tercer orden que presentaron los participantes. Con la finalidad de eliminar todos los sustantivos de uso frecuente en la lengua, para asegurarnos de que solo se incluyeran vocablos complejos y más especializados, se procedió a eliminar todos aquellos sustantivos que aparecieran en el Diccionario del vocabulario fundamental de México (Lara, 2013). Se eligió este procedimiento porque dicho diccionario contiene la lista de los vocablos que están presentes en el 75\% de todas las emisiones verbales de los mexicanos. Una vez que se había descartado que los sustantivos de tercer orden empleados por los participantes no fueran parte del uso frecuente de la lengua, se podría afirmar que se trataba de un léxico de tipo más académico literario. La Tabla 6 presenta el listado de las palabras encontradas bajo ese criterio para ambos grupos de adolescentes en ambas modalidades.

Los datos de la Tabla 6 muestran, en primera instancia, una enorme diferencia en el uso de sustantivos de tercer orden entre grupos. Mientras que los adolescentes del Grupo 1 presentan una amplia variedad de este tipo de sustantivos en ambas modalidades (aunque más en la escritura), fueron muy poco frecuentes en las narraciones de los jóvenes del Grupo 2. Además, se observa que para ambos grupos la mayor cantidad de palabras diferentes se concentra en la categoría de los sustantivos del nivel 10 (abstractos derivados de otras categorías de palabras), lo que corresponde a los sustantivos más complejos. A la vez, es importante observar cómo hay un mayor uso de este tipo de sustantivos en la lengua escrita que en la lengua oral para todos los jóvenes. Por último, resulta esencial destacar que todos los sustantivos del nivel 10 corresponden a nominalizaciones. Nuevamente encontramos que los adolescentes del Grupo 1 parecen tener una ventaja sobre los jóvenes del Grupo 2 en la producción de este tipo de sustantivos.

\section{Análisis de expresiones no literales en las narraciones}

Por último, se decidió realizar un análisis más de tipo cualitativo de los usos que hicieron los adolescentes de las expresiones no literales en sus narraciones orales y escritas. Si bien no todas estas expresiones se basan en un uso no literal de los sustantivos por sí mismos, la inclusión de expresiones no literales incluye un uso particular de los sustantivos y corresponde al campo de la elección léxica, por lo que su estudio resultó pertinente para nuestro trabajo.

En términos generales el análisis de las expresiones no literales señaló diferencias importantes entre grupos. Así, se observó que el uso de expresiones no literales fue mucho más frecuente en la lengua escrita que en la oral solo en el caso del Grupo 1 ( 7 expresiones en la oralidad y 42 en la escritura), en tanto que el Grupo 2 empleó una cantidad similar de expresiones no literales en la oralidad y la escritura ( 9 en las narraciones orales y 10 en las escritas). Otra diferencia importante entre grupos radicó en el tipo de expresiones incluidas en los relatos. Los jóvenes del Grupo 2 utilizaron únicamente expresiones no literales 
Tabla 6

Sustantivos de Tercer Orden de Uso Poco Frecuente Presentes en las Narraciones Orales y Escritas de los

Participantes de Ambos Grupos

\begin{tabular}{|c|c|c|c|c|}
\hline \multirow{2}{*}{ Tipo de sustantivo } & \multicolumn{2}{|c|}{ Grupo 1} & \multicolumn{2}{|c|}{ Grupo 2} \\
\hline & Oral & Escrito & Oral & Escrito \\
\hline $\begin{array}{l}\text { Nivel } 9 \\
\text { Sustantivos abstractos no imaginables } \\
\text { poco individuados }\end{array}$ & $\begin{array}{l}\text { creciente } \\
\text { ganas } \\
\text { mente }\end{array}$ & $\begin{array}{l}\text { creciente } \\
\text { instante } \\
\text { mente } \\
\text { sonido } \\
\text { tinte }\end{array}$ & oxígeno & \\
\hline $\begin{array}{l}\text { Nivel } 10 \\
\text { Sustantivos abstractos derivados }\end{array}$ & $\begin{array}{l}\text { daño } \\
\text { esperanza } \\
\text { sentimiento }\end{array}$ & $\begin{array}{l}\text { anchura } \\
\text { angustia } \\
\text { ansias } \\
\text { asombro } \\
\text { caudal } \\
\text { costumbre } \\
\text { culpa } \\
\text { desesperación } \\
\text { detalle } \\
\text { esperanza } \\
\text { furia } \\
\text { impresión } \\
\text { incomodidad } \\
\text { lesión } \\
\text { melancolía } \\
\text { pensamiento } \\
\text { sensación } \\
\text { tensión } \\
\text { tristeza }\end{array}$ & recuerdo & $\begin{array}{l}\text { elección } \\
\text { riesgo } \\
\text { vitalidad }\end{array}$ \\
\hline
\end{tabular}

que ocurren frecuentemente en contextos coloquiales conversacionales, como se observa en los siguientes ejemplos:

"pero nunca se me pasó por aquí por la cabeza que se había muerto mi papá" (AM, mujer, 12, oral, G2)

"como que sí me entró miedo" (A, hombre, 14, oral, G2)

"lo material da igual" (A, mujer, 14, escrito, G2)

"ya estábamos embarrados en el poste" (I, hombre, 16, escrito, G2)

En cambio, los adolescentes del Grupo 2 emplearon, sobre todo en sus narraciones escritas, expresiones no literales propias de un genéro más literario. Obsérvense los siguientes ejemplos:

"se respiraba tristeza y melancolía" (K, mujer, 12 , escrita, G1)

"una sensación de frío me recorría toda la espalda" (M, mujer, 14, escrita, G1)

"el dolor me obligaba a cojear" (A, hombre, 14, escrita, G1)

"era demasiado tarde" (lit. no había remedio) (G, hombre, 16, escrita, G1)

Por otro lado, cuando los jóvenes del Grupo 1 usaban las mismas expresiones no literales en sus 
narraciones orales y escritas, tendían a presentar mayor complejidad en la escritura:

"dimos tres vueltas de campana en el coche" (E, hombre, 12, oral, G1)

"en el momento cumbre el coche desestabilizado después de un salto dio tres bruscas, largas y tenebrosas vueltas de campana" (E, hombre, 12, escrita, G1)

"y ya de suerte no pasó nada" (L, mujer, 16, oral, G1)

"y ya de suerte no pasó del susto" (L, mujer, 16, escrita, G1)

En cambio, los adolescentes del Grupo 2 emplearon expresiones muy similares en la oralidad y la escritura:

"y pues ya ni le tomaba importancia" (C, hombre, 14, oral, G2)

"pero ya no le tomé importancia" (C, hombre, 14, escrita, G2)

"la única experiencia así muy fuerte que he tenido" (AP, mujer, 16, oral, G2)

"fue una experiencia fuerte" (AP, mujer, 16, escrita, G2)

A la vez, una gran cantidad de las expresiones no literales usadas por los adolescentes del Grupo 1, y no así las del Grupo 2, tenían la intención de evaluar la narración completa y marcar la razón por la cual el relato había tenido lugar, como se observa en el siguiente ejemplo:

"me di cuenta de lo frágil que es la vida, de lo inesperada que es la muerte, pero sobre todo me di cuenta de que un hombre por más diminuto que parezca puede hacer la diferencia entre una muerte segura y una vida llena de alegría" (P, hombre, 12, escrita, G1)

Por otra parte, se observó que ambos grupos de adolescentes incluyeron expresiones no literales para la mención a estados mentales, intenciones, emociones y procesos metacognitivos, aunque esto solo en las narraciones escritas y no en las orales:

"a todos se nos vino a la mente" (G, hombre, 14, escrita, G1, estado mental)

"estaba a punto de cruzar una calle" (M, mujer, 14, escrita, G1, intención)

"el aire se volvió pesado" (P, hombre, 12, escrita, G1, emoción)

"algo interrumpió mis pensamientos" (K, mujer, 12, escrita, G1, proceso metacognitivo)

No obstante, es interesante mencionar que mientras que los adolescentes del Grupo 1 hicieron un uso variado de este recurso lingüístico, las únicas dos expresiones no literales para señalar procesos cognitivos en los jóvenes del Grupo 2 fueron combinaciones con tener idea de, de índole muy coloquial, como se ve a continuación:

"yo no tenía idea de lo que pasaba con él" (AM, mujer, 12, escrita, G2, estado mental)

"mi muchacha no tenía idea de qué hacer" (C, hombre, 12, escrita, G2, estado mental)

\section{Discusión}

A lo largo de esta investigación se propuso observar la manera en que adolescentes de 12, 14 y 16 años pertenecientes a dos entornos sociales diferentes (con mayor y menor acceso a la cultura escrita) hacen una elección léxica (uso diferenciado de sustantivos) dependiendo de la tarea a la que se enfrentan (narración oral o escrita). Los datos obtenidos señalan usos diferentes de los sustantivos dependiendo de la modalidad discursiva (narración oral vs. escrita) y el grupo. De esta manera hubo, como era de esperarse, un uso más frecuente de sustantivos propios de un lenguaje más académico literario en los textos escritos y en aquellos producidos 
por los adolescentes que pertenecen en mayor medida a una cultura escrita.

En cuanto a las diferencias debidas a la modalidad oral/escrito, los resultados indican que la lengua escrita incide en la presencia de una mayor cantidad de sustantivos por cláusula (densidad nominal), así como en el uso de sustantivos de índole más compleja (de segundo y tercer órdenes). En el caso de los sustantivos de tercer orden, además se observa que la lengua escrita favorece en gran medida la producción de sustantivos abstractos derivados de otras categorías gramaticales, es decir, de sustantivos producto de la nominalización (sustantivos de nivel 10). Adicionalmente, los hallazgos también señalan que la lengua escrita lleva a la aparición de expresiones no literales y sobre todo de aquellas empleadas para la expresión de estados mentales, intenciones, emociones y procesos metacognitivos. Todo esto coincide con los resultados señalados por otros autores en torno a la importancia de la lengua escrita para el desarrollo de un vocabulario más complejo y especializado (véase Beck, McKeown \& Kucan, 2008; Berman \& Ravid, 2010; Nippold, 2007; Ramachandra \& Karanth, 2007; Ravid, 2006; Ravid \& Cahana-Amitay, 2008; Snow \& Uccelli, 2009, entre otros).

Por otra parte, en lo que se refiere a la comparación entre grupos de adolescentes, los resultados mostraron un comportamiento similar para ambos grupos en algunos aspectos. En primera instancia, los jóvenes de las dos escuelas presentaron una mayor densidad nominal en sus narraciones escritas que en las orales. A su vez, los tipos de sustantivos más favorecidos por los adolescentes fueron los de primer orden, seguidos por los de segundo y tercer orden, respectivamente.
Los hallazgos también señalan diferencias importantes en el uso que los jóvenes de los dos grupos le dan a los sustantivos. De esta manera, se observa un uso más diferenciado de estos dependiendo de la modalidad (oral/escrito) en los adolescentes del Grupo 1. Así, estos presentaron una proporción distinta de sustantivos de primer, segundo y tercer orden dependiendo de si sus relatos eran orales o escritos, lo que habla de una mayor capacidad de elección léxica. Además, emplearon sustantivos y expresiones no literales más complejos en la escritura que en la oralidad. Finalmente, los adolescentes del Grupo 1 mostraron mayor cantidad y variedad de sustantivos complejos (tercer orden) en sus relatos, así como más expresiones no literales para describir estados mentales y para evaluar las narraciones completas que los jóvenes del Grupo 2. Estos resultados nos llevan a señalar que las experiencias que los adolescentes del Grupo 1 han tenido con la lengua escrita tanto en la escuela como en el hogar han repercutido de manera importante tanto en la adquisición de un vocabulario más complejo como en la capacidad para elegir el vocabulario más adecuado dependiendo del contexto al que se enfrentan. Esto, a su vez, confirma la importancia del acceso a la lengua escrita durante los años escolares en la adquisición de las habilidades lingüísticas.

Si bien los resultados de este estudio permiten encontrar diferencias debidas al entorno social de los individuos en el uso del léxico académico literario en narraciones, queda por investigar si la pertenencia a una cultura escrita también incide en el desarrollo de la elección léxica en otros tipos de textos más académicos o escolarizados, como podrían ser los científicoexpositivos. Lo anterior queda pendiente para futuras investigaciones. 


\section{Referencias}

Alvarado, M., Calderón, G., Hess, K \& Vernon, S. (2011). Historia y perspectivas teóricas sobre la adquisición de la lengua oral y escrita en los años escolares. En K. Hess, G. Calderón, S. Vernon, \& M. Alvarado (Coords.). Desarrollo lingüístico y cultura escrita: puntos, acentos, historias, metáforas y argumentos (pp. 5-26). México: Porrúa-UAQ.

Barriga, R. (2002). Estudios sobre habla infantil en los años escolares: un solecito calientote. México: El Colegio de México.

Barton, D. (2007). Literacy: An Introduction to the Ecology of Written Language. Oxford: Blackwell.

Baumann, J. F. (2008). Vocabulary and reading comprehension: the nexus of meaning. En S. E. Israel \& G. G. Duffy (Eds.), Handbook of Research on Reading Comprehension (pp. 323-34). Nueva York: Routledge.

Beck, I. L., McKeown, M. G. \& Kucan, L. (2008). Creating Robust Vocabulary. Nueva York: The Guilford Press.

Berman, R. A. (2004a). Between emergence and mastery. The long developmental route of language acquisition. En R. A. Berman (Ed.), Language Development across Childhood and Adolescence (pp. 9-34). Amsterdam-Philadelphia: John Benjamins.

Berman, R. A. (2004b). The role of context in developing narrative abilities. En S. Strömqvist \& L. Verhoeven (Eds.), Relating Events in Narrative: Typological and Contextual Perspectives (pp. 261-280). Mahwah NJ: Lawrence Erlbaum.

Berman, R. A. \& Ravid, D. (2010). Developing noun phrase complexity at school age: a text-embedded cross-linguistic analysis. First Language, 30, 3-26.

Berman, R. A. \& Slobin, D. I. (1994). Relating Events in Narrative. Hillsdale NJ: Lawrence Erlbaum.

Calderón, G. (2012). La comprensión de la metáfora en niños y jóvenes: el caso de las adivinanzas. (Tesis inédita de doctorado). Universidad Autónoma de Querétaro, México.
Carmiol, A., Ríos, M. \& Sparks, A. (en prensa). La relación entre habilidades prelectoras y habilidades narrativas en niños y niñas preescolares costarricenses: aportes para un enfoque comprensivo de la alfabetización emergente. En A. Auza \& K. Hess (Eds.), ¿Qué me cuentas? Narraciones y desarrollo lingüistico en niños hispanohablantes. México: Universidad Autónoma de Querétaro-Hospital Dr. Manuel Gea González.

Cassany, D., Luna, M., \& Sanz, G. (1994). Enseñar lengua. Barcelona: Graó.

Dockrell, J. \& Messer, D. (2004). Lexical acquisition in the early school years. En R. A. Berman (Ed.), Language Development Across Childhood and Adolescence (pp. 35-52). Amsterdam-Philadelphia: John Benjamins.

Gombert, J. (1992). Metalinguistic Development. Chicago: University of Chicago Press.

Hickmann, M. (2003). Children's Discourse: Person, Space and Time Across Languages. Cambridge: Cambridge University Press.

Hess, K. (2010). Saber lengua: lenguaje y metalenguaje en los años escolares. México: El Colegio de México.

Hyland, K. (2005). Metadiscourse: Exploring Interaction in Writing. Nueva York: Continuum.

Irvin, J. L. (1990). Reading and the Middle School Student. Boston: Allyn and Bacon.

Jisa, H. (2004). Growing into academic French. En R. A. Berman (Ed.), Language Development Across Childhood and Adolescence (pp. 135-161). Amsterdam-Philadelphia: John Benjamins.

Labov, W. \& Waletzky, J. (1997). Narrative analysis: oral versions of personal experience. Journal of Narrative and Life History, 7, 3-38.

Lara, L. F. (2013). Vocabulario fundamental del español. Recuperado de: http://dem.colmex.mx/ repository/pdfs/vocabulario-fundamental.pdf

Larsen, J. A. \& Nippold, M. A. (2007). Morphological analysis in school-age children: dynamic assessment of a word learning strategy. Language, Speech, and Hearing Services in Schools, 38, 201-212. 
MacWhinney, B. (1991). The CHILDES Project: Tools for Analyzing Talk. Hillsdale NJ: Lawrence Erlbaum.

Mancilla-Martínez, J. \& Lesaux, N. K. (2011). Early home language use and later vocabulary development. Journal of Educational Psychology, Advance online publication. doi: 10.1037/ a0023655.

Melzi, G. \& Ely, R. (2010). Lenguaje y lectoescritura en los años escolares. En J. Berko Gleason \& N. Bernstein Ratner (2010). El desarrollo del lenguaje (pp. 413-459). Madrid: Pearson.

Mostacero, R. (2004). Construcción de la escritura personal a partir del discurso del otro. Lingua Americana, 15, 63-79.

Nippold, M. (2007). Later Language Development: School-Age Children, Adolescents, and Young Adults. Austin TX: Pro-Ed.

Nippold, M. A., \& Sun, L. (2008). Knowledge of morphologically complex words: a developmental study of older children and young adolescents. Language, Speech, and Hearing Services in Schools, 39, 365-373.

Pence, K. L. \& Justice, L. M. (2008). Language Development from Theory to Practice. Upper Saddle River NJ: Pearson-Prentice Hall.

Ramachandra, V. \& Karanth, P. (2007). The role of literacy in the conceptualization of words: data from Kannada-speaking children and non-literate adults. Reading and Writing, 20, 173-199.
Ravid, D. (2006). Semantic development in textual contexts during the school years: Noun Scale analyses. Journal of Child Language, 33, 791-821.

Ravid, D. (2011). Spelling Morphology: The Psycholinguistics of Hebrew Spelling. New York: Springer.

Ravid, D. \& Cahana-Amitay, D. (2005). Verbal and nominal expressions in narrating conflict situations in Hebrew. Journal of Pragmatics, 37, 157183.

Schleppegrell, M. J. (2004). The Language of Schooling: A Functional Linguistics Perspective. Mahwah NJ: Lawrence Erlbaum.

Snow, C. E. \& Uccelli, P. (2009) The challenge of academic language. En D. Olson \& N. Torrance (Eds.). Cambridge Handbook of Literacy (pp. 112133). Cambridge: Cambridge University Press.

Tolchinsky, L. (2004). The nature and scope of later language development. En R. A. Berman (Ed.). Language Development Across Childhood and Adolescence (pp. 9-34). Amsterdam-Philadelphia: John Benjamins.

Tolchinsky, L., Rosado, E., Aparici, M. \& Perera, J. (2005). Becoming proficient educated users of language. En D. Ravid. D. \& H. Bat-Zeev Shyldkrot (Eds.). Perspectives on Language and Language Development: Essays in Honor of $\mathrm{Ruth} A$. Berman (pp. 375-389). Dordrecht: Kluwer.

Zwiers, J. (2008). Building Academic Language: Essential Practices for Content Classrooms. San Francisco: Jossey-Bass. 